\title{
RESEARCH
}

Open Access

\section{Apoptotic bodies derived from mesenchymal stem cells promote cutaneous wound healing via regulating the functions of macrophages}

Jin Liu ${ }^{1,2+}$, Xinyu Qiu ${ }^{1+}$, Yajie Lv ${ }^{1,3+}$, Chenxi Zheng ${ }^{1}$, Yan Dong ${ }^{2}$, Geng Dou ${ }^{1}$, Bin Zhu ${ }^{1,4}$, Anqi Liu', Wei Wang ${ }^{5}$, Jun Zhou', Siying Liu', Shiyu Liu", Bo Gao ${ }^{2^{*}}$ and Yan Jin ${ }^{1^{*}}$ (D)

\begin{abstract}
Background: As the major interface between the body and the external environment, the skin is liable to various injuries. Skin injuries often lead to severe disability, and the exploration of promising therapeutic strategies is of great importance. Exogenous mesenchymal stem cell (MSC)-based therapy is a potential strategy due to the apparent therapeutic effects, while the underlying mechanism is still elusive. Interestingly, we observed the extensive apoptosis of exogenous bone marrow mesenchymal stem cells (BMMSCs) in a short time after transplantation in mouse skin wound healing models. Considering the roles of extracellular vesicles (EVs) in intercellular communication, we hypothesized that the numerous apoptotic bodies (ABs) released during apoptosis may partially contribute to the therapeutic effects.
\end{abstract}

Methods: ABs derived from MSCs were extracted, characterized, and applied in mouse skin wound healing models, and the therapeutic effects were evaluated. Then, the target cells of ABs were explored, and the effects of ABs on macrophages were investigated in vitro.

Results: We found ABs derived from MSCs promoted cutaneous wound healing via triggering the polarization of macrophages towards M2 phenotype. In addition, the functional converted macrophages further enhanced the migration and proliferation abilities of fibroblasts, which together facilitated the wound healing process.

(Continued on next page)

\footnotetext{
*Correspondence: liushiyu@vip.163.com; gaobo@fmmu.edu.cn; yanjin@fmmu.edu.cn

†in Liu, Xinyu Qiu and Yajie Lv contributed equally to this work. ${ }^{1}$ State Key Laboratory of Military Stomatology\& National Clinical Research Center for Oral Diseases \& Shaanxi International Joint Research Center for Oral Diseases, Center for Tissue Engineering, School of Stomatology, The Fourth Military Medical University, Xi'an 710032, Shaanxi, China

${ }^{2}$ State Key Laboratory of Military Stomatology \& National Clinical Research Center for Oral Diseases \& Shaanxi Key Laboratory of Stomatology,

Department of Prosthodontics, School of Stomatology, The Fourth Military Medical University, Xi'an 710032, Shaanxi, China

Full list of author information is available at the end of the article
}

(c) The Author(s). 2020 Open Access This article is licensed under a Creative Commons Attribution 4.0 International License, which permits use, sharing, adaptation, distribution and reproduction in any medium or format, as long as you give appropriate credit to the original author(s) and the source, provide a link to the Creative Commons licence, and indicate if changes were made. The images or other third party material in this article are included in the article's Creative Commons licence, unless indicated otherwise in a credit line to the material. If material is not included in the article's Creative Commons licence and your intended use is not permitted by statutory regulation or exceeds the permitted use, you will need to obtain permission directly from the copyright holder. To view a copy of this licence, visit http://creativecommons.org/licenses/by/4.0/ The Creative Commons Public Domain Dedication waiver (http://creativecommons.org/publicdomain/zero/1.0/) applies to the data made available in this article, unless otherwise stated in a credit line to the data. 
(Continued from previous page)

Conclusions: Collectively, our study demonstrated that transplanted MSCs promoted cutaneous wound healing partially through releasing apoptotic bodies which could convert the macrophages towards an antiinflammatory phenotype that plays a crucial role in the tissue repair process.

Keywords: Apoptotic bodies, Extracellular vesicles, Macrophage polarization, Skin regeneration

\section{Introduction}

The skin is the largest organ of the human body which serves as the first defense line to the external environment, so that it is liable to different kinds of injuries. Large area loss of the skin often leads to severe disability or even death, which not only physically and mentally suffers the patients, but also brings a heavy burden to the society [1]. To date, various strategies have been explored, among which exogenous mesenchymal stem cells (MSCs) showed a great potential [2]. More and more studies have demonstrated that MSCs could accelerate cutaneous wound healing and restore the more integrate and organized skin structure [3-5]. However, the underlying mechanism remains elusive. Nowadays, paracrine pathway is increasingly approved, by which MSCs release growth factors, cytokines, and extracellular vesicles beneficial to the wound healing process [6-8]. Interestingly, we previously observed extensive apoptosis of transplanted MSCs in a short time after transplantation in a rabbit skin wound healing model and found that apoptosis has an important role in the activation of the inflammatory regulatory abilities of MSCs [9]. Likewise, using a murine model of graft-versus-host disease (GvHD), another study demonstrated that MSCs undergo extensive caspase activation and apoptosis after transplantation, which is necessary for their immunosuppressive function [10].

Apoptosis refers to the programmed cell death, which is of great importance in the development, growth, and immune processes of organisms [11]. During apoptosis, cells undergo a series of morphological changes and eventually form apoptotic bodies (ABs) [12]. ABs are the largest kind of extracellular vesicles with a rich cargo like DNA, RNA, proteins, and organelles [13]. As it contains multiple contents, we supposed that $\mathrm{ABs}$ could contribute to the therapeutic effects of MSCs after transplantation. Moreover, once apoptosis occurs, apoptotic signals are transmitted promptly to phagocytes, so that apoptotic cells and debris can be cleared in a short time [14]. Macrophages are the professional phagocytes which are responsible for the clearance of apoptotic issues [15]. Meanwhile, macrophages are important immune cells, which play essential roles both in inflammation and tissue repair processes [16]. Thereby, we inferred that macrophages are the target cells of ABs.

In this study, we verified the therapeutic effects of transplanted MSCs in mouse skin wound healing models and observed vast apoptosis of transplanted MSCs in a short time. Then, we extracted ABs and utilized them to demonstrate that $\mathrm{ABs}$ could accelerate the cutaneous wound healing process. Meanwhile, we found that macrophages were probably the target cells of $A B s$ both in vivo and in vitro experiments and proved that $A B s$ could promote macrophage polarization towards the M2 phenotype. Finally, M2 phenotypic macrophages further enhanced the function of fibroblasts, which synergistically promoted cutaneous wound healing. In conclusion, we revealed the underlying mechanism for exogenous MSC-based therapy and provided a new therapeutic strategy for skin injuries.

\section{Materials and methods Animals}

All procedures were approved by the Institutional Animal Care and Use Committee of the Fourth Military Medical University and conformed to the Guide for the Care and Use of Laboratory Animals published by the National Institutes of Health. All animals were purchased from the Laboratory Animal Center of the Fourth Military Medical University. Eight-week-old female wild type (WT) C57BL/6 mice were used both for isolation of cells and establishment of skin wound healing models. The mice were kept under specific pathogen-free conditions $\left(24^{\circ} \mathrm{C}, 12\right.$-h light/dark cycles, and $50 \%$ humidity) with free access to food and water.

\section{Cell culture}

Isolation and culture of murine bone marrow mesenchymal stem cells (BMMSCs) were performed as previously described [17]. Eight-week-old female WT C57BL/6 mice (18-20 g) were used. The hindlimbs were removed, and then bone marrow cells were separated by flushing marrow cavities and seeded in a medium of alphaminimum essential medium ( $\alpha$-MEM) (Gibco, USA) supplemented with $20 \%$ fetal bovine serum (FBS) (Gibco, USA), $2 \mathrm{mM} \mathrm{L}$-glutamine, $100 \mathrm{U} / \mathrm{ml}$ penicillin, and $100 \mathrm{~g} /$ $\mathrm{ml}$ streptomycin (all from Invitrogen, USA). For in vivo tracing, BMMSCs were pre-labeled with PKH26 (SigmaAldrich, USA) according to the manufacturers' protocol and detected by the confocal microscope (Nikon, Japan).

Bone marrow-derived monocytes (BMDMs) were also isolated from hindlimbs. Bone marrow cells were separated and washed in Red Blood Cell Lysis Buffer (Beyotime, China). The cell suspension was centrifuged at $100 g$ for 5 min and then the cell pellet was resuspended and seeded in a medium of high-glucose Dulbecco's modified Eagle 
medium (DMEM) (Gibco, USA) supplemented with 10\% FBS, $2 \mathrm{mM}$ L-glutamine, $100 \mathrm{U} / \mathrm{ml}$ penicillin, and $100 \mathrm{~g} / \mathrm{ml}$ streptomycin. Cells were cultured for 7 days in the presence of recombinant mouse macrophage colony-stimulating factor (M-CSF) (R\&D, USA) at $20 \mathrm{ng} / \mathrm{ml}$ to induce mature macrophages. After 7 days, macrophages were added with lipopolysaccharide (LPS) (Sigma-Aldrich, USA) at $1 \mu \mathrm{g} / \mathrm{ml}$ for $24 \mathrm{~h}$ to stimulate inflammation.

Primary fibroblasts were isolated from neonatal murine skin tissue. After enzymatic digestion in dispase (Gibco, USA) overnight at $4{ }^{\circ} \mathrm{C}$, the dermis was separated from the epidermis. Then, the dermis was minced into small pieces and incubated in type I collagenase (Gibco, USA) for $1 \mathrm{~h}$ at $37^{\circ} \mathrm{C}$ with manual agitation every $15 \mathrm{~min}$. The cell suspension was filtrated with a 100-mesh sieve and centrifuged at $100 \mathrm{~g}$ for $5 \mathrm{~min}$. The cell pellet was resuspended and seeded in a medium of $\alpha$-MEM supplemented with $10 \%$ FBS, $2 \mathrm{mM}$ L-glutamine, $100 \mathrm{U} / \mathrm{ml}$ penicillin, and $100 \mathrm{~g} / \mathrm{ml}$ streptomycin.

All cells were cultured at $37^{\circ} \mathrm{C}$ in a humidified atmosphere of $5 \% \mathrm{CO}_{2}$. Cells were digested and passaged by using $0.25 \%$ trypsin (Invitrogen, USA) at $80-90 \%$ confluence.

\section{Isolation and characterization of apoptotic bodies}

Apoptotic bodies (ABs) were isolated by using the optimized protocol. Firstly, BMMSCs were treated with staurosporine (STS) (Cell Signaling Technology, USA) at $0.5 \mu \mathrm{M}$ for $12 \mathrm{~h}$ to induce apoptosis. The supernatant was collected and centrifuged at $1000 \mathrm{~g}$ for $10 \mathrm{~min}$ at $4{ }^{\circ} \mathrm{C}$ to remove cells and debris. Then, the supernatant was further centrifuged at $16000 \mathrm{~g}$ for $30 \mathrm{~min}$ at $4{ }^{\circ} \mathrm{C}$, and the pellet was washed twice in phosphate-buffered saline (PBS). The isolated ABs were suspended in $100 \mu \mathrm{l}$ PBS and stored at $-80^{\circ} \mathrm{C}$ for further study if necessary. ABs were quantified by BCA assay (TIANGEN, China) before using. The morphological features of ABs were observed by a scanning electron microscopy (SEM) (Hitachi, Japan), and the size distribution was measured by dynamic light scattering (DLS) analysis by using Zetasizer Nano ZSE (Malvern, $\mathrm{UK})$. The expression of $\mathrm{C} 1 \mathrm{q}$ and Annexin $\mathrm{V}$ in $\mathrm{ABs}$ were examined by immunofluorescent staining. The Annexin $\mathrm{V}$ staining was carried out by using an Annexin V-FITC apoptosis assay kit (7Sea Biotech, China). The protein expression levels of caspase- 3 in BMMSCs and ABs were detected by western blots. To detect phagocytosis both in vivo and in vitro, ABs were labeled with PKH26 and detected by a confocal microscope. The primary antibodies involved in this study include C1q (1:200) (CEDARLANE, CL7501F, monoclonal, Canada), $\beta$-actin (1:1000) (CWBio, CW0096, monoclonal, China), and caspase-3 (1:1000) (Cell Signaling Technology, 9662, polyclonal, USA).

\section{Flow cytometric analysis of cell phenotype}

For flow cytometric analysis of the cell surface markers, MSCs were detached by using $0.25 \%$ trypsin and resuspended in PBS supplemented with 3\% FBS. Then, the cells were incubated in dark at $4{ }^{\circ} \mathrm{C}$ for $30 \mathrm{~min}$ with phycoerythrin (PE)-conjugated mouse anti-CD29 (460299-41, monoclonal), anti-CD34 (15-0349-41, monoclonal), anti-CD105 (12-1057-42, monoclonal), anti-CD146 (12-1469-42, monoclonal) and fluorescein isothiocyanate (FITC)-conjugated mouse anti-CD11b (11-0112-82, monoclonal), anti-CD45 (11-0451-82, monoclonal), anti-CD90 (11-0900-81, monoclonal), and anti-Sca-1 (11-5981-82, monoclonal) (all from eBioscience, USA), respectively. Related conjugated IgG (eBioscience, USA) was used as the negative control. Finally, cells were washed twice in PBS and positively stained cells were detected by the flow cytometer (Beckman Coulter, USA).

\section{Osteogenic differentiation assay}

MSCs were seeded in six-well plates at a density of $5 \times$ $10^{5}$ cells per well. When cells reached $100 \%$ confluence, the basal medium was changed into osteogenic induction medium: $\alpha$-MEM containing $10 \%$ FBS, $1 \%$ penicillin/streptomycin, $5 \mathrm{mM} \beta$-glycerophosphate, $50 \mu \mathrm{g} / \mathrm{ml}$ ascorbic acid, and $10 \mathrm{nM}$ dexamethasone (all from Sigma-Aldrich, USA). The medium was refreshed every other day. After 7 days of osteogenic induction, alkaline phosphatase (ALP) staining was performed with the commercial kit (Beyotime, China). After 21 days of osteogenic induction, Alizarin red staining was performed. Photographs were taken by using an inverted optical microscope (Olympus, Japan).

\section{Adipogenic differentiation assay}

MSCs were seeded in six-well plates at a density of $5 \times 10^{5}$ cells per well. When cells reached $100 \%$ confluence, the basal medium was changed into adipogenic induction medium: $\alpha$ MEM containing 10\% FBS, $0.5 \mathrm{mM}$ isobutylmethylxanthine, $0.5 \mathrm{mM}$ dexamethasone, and $60 \mathrm{nM}$ indomethacin (all from Sigma-Aldrich, USA). The medium was refreshed every other day. After 7 days of adipogenic induction, Oil Red O staining was performed to indicate lipid droplet formation. Photographs were taken by using an inverted optical microscope (Olympus, Japan).

\section{Colony-forming unit assays}

MSCs were seeded in 5-cm culture dishes at a density of $1 \times 10^{4}$ cells per dish and cultured in basal medium for 2 weeks. The medium was refreshed every 3 days until colonies with over 50 cells were observed. The colonies were stained with $0.2 \%$ crystal violet (Sigma-Aldrich, USA), and photographs were taken by using an inverted optical microscope (Olympus, Japan).

\section{Skin wound healing models}

Eight-week-old female WT C57BL/6 mice (18-20 g) were used. Thirty-six mice underwent anesthesia by an 
intraperitoneal injection of pentobarbitone sodium (40 $\mathrm{mg} / \mathrm{kg}$ ). After shaving and cleaning, a full-thickness wound $(1 \mathrm{~cm}$ in diameter) was created on the dorsal skin. The mice were randomly divided into four groups: PBS group, Gel group, Gel+MSCs group, and Gel+ABs group. Commercial hydrogel Pluronic F-127 (PF-127) (Sigma-Aldrich, USA) was dissolved in PBS at a concentration of $30 \%$ at $4{ }^{\circ} \mathrm{C}$. MSCs and ABs were embedded in PF-127 solution at $4{ }^{\circ} \mathrm{C}$. The number of MSCs was $2 \times$ $10^{6}$ (in $100 \mu \mathrm{l}$ PF-127 solution) when they were applied to the wound site, which was calculated by the digital cell counter (Bio-Rad, USA). The amount of ABs was $50 \mu \mathrm{g}$ (in $100 \mu \mathrm{l}$ PF-127 solution) when they were applied to the wound site, which was measured by BCA assay (TIANGEN, PA115). The treatments were conducted on days 0,3 , and 7 , and the dressings were kept on the wound site until the next time point. After the operation, the wound was covered by surgical dressings ( $3 \mathrm{M}$, USA) and the mice were housed individually. The wound area and body weight were measured on days 0 , $3,7,10$, and 14 post-surgery, and the wound healing rate was calculated according to the formula: $\mathrm{D}_{n}$ wound healing rate $=\left(D_{0}\right.$ wound area $-D_{n}$ wound area $) / D_{0}$ wound area $\times 100 \%$. The mice were sacrificed on day 14 , and the skin tissues were harvested for further analysis.

\section{Immunofluorescent staining}

Cells and skin tissue samples were fixed in $4 \%$ paraformaldehyde overnight. Skin tissue samples underwent dehydration with 30\% saccharose and were embedded in optimal cutting temperature compound (OCT) (Leica, Germany) and cut into 10- $\mu \mathrm{m}$-thick sections. The cells and sections underwent permeabilization with $0.05 \%$ Triton X-100 (Sigma-Aldrich, USA) for $10 \mathrm{~min}$ at room temperature, blockage with $5 \%$ bovine serum albumin (BSA) (Sigma-Aldrich, USA) at $37^{\circ} \mathrm{C}$ for $30 \mathrm{~min}$, and incubation with the primary antibody overnight at $4{ }^{\circ} \mathrm{C}$. Next, the sections were incubated with fluorescence secondary antibody (Sigma-Aldrich, USA) at room temperature for $1 \mathrm{~h}$. Lastly, the nuclei were counterstained by Hoechst 33342 (Sigma-Aldrich, USA) for $10 \mathrm{~min}$ at room temperature. Photographs were taken by a confocal microscope and evaluated by ImageJ software. The primary antibodies involved in this study include F4/80 (1:200, ab6640, monoclonal), Cytokeratin 14 (1:400, ab181595, monoclonal), CD206 (1:200, ab195191, monoclonal), and Ki67 (1:150, ab16667, monoclonal) (all from Abcam, UK).

\section{Histological staining}

Skin tissue samples were fixed in $4 \%$ paraformaldehyde for $24 \mathrm{~h}$ and underwent dehydration with graded ethanol. Then, the samples were embedded in paraffin and cut into $4-\mu \mathrm{m}$-thick sections. H\&E staining and Masson staining were carried out by using the commercial staining kits (Baso technology, China).

\section{Cell migration assay}

Fibroblasts were seeded in 6-well plates at a density of $5 \times$ $10^{5}$ cells per well. When cells reached $100 \%$ confluence, the scratch was made on the plates by using sterile pipette tips. Cells were cultured in medium cocultured with $\mathrm{ABs}$ at $5 \mu \mathrm{g} / \mathrm{ml}, 10 \mu \mathrm{g} / \mathrm{ml}, 20 \mu \mathrm{g} / \mathrm{ml}$, and $30 \mu \mathrm{g} / \mathrm{ml}$ respectively, or conditioned medium collected from BMDM. Cells cultured in serum-free medium were used as the negative control. Photographs were taken by an inverted microscope at $0 \mathrm{~h}$, $12 \mathrm{~h}$, and $24 \mathrm{~h}$ and evaluated by ImageJ software.

\section{RNA extraction and qPCR}

BMDMs were treated with $\mathrm{ABs}$ at $5 \mu \mathrm{g} / \mathrm{ml}, 10 \mu \mathrm{g} / \mathrm{ml}$, $20 \mu \mathrm{g} / \mathrm{ml}$, and $30 \mu \mathrm{g} / \mathrm{ml}$ respectively. The culture medium was discarded, and the total RNA of BMDMs was extracted by using Trizol Reagent (Takara, Japan) $24 \mathrm{~h}$ later. After that, complementary DNA (cDNA) was synthesized using a PrimeScript RT reagent kit (Takara, Japan). Then, qPCR procedure was performed with CFX96TM system (Bio-Rad, USA) using a SYBR Premix Ex Taq II kit (Takara, Japan). Gapdh was used as the internal control. All the primer sequences are listed in Table 1.

\section{ELISA}

Culture medium from BMDMs was collected after treatment with $\mathrm{ABs}$ at $5 \mu \mathrm{g} / \mathrm{ml}, 10 \mu \mathrm{g} / \mathrm{ml}, 20 \mu \mathrm{g} / \mathrm{ml}$, and $30 \mu \mathrm{g} / \mathrm{ml}$ respectively. To exclude the production of TGF- $\beta$ and/or IL- 10 by ABs themselves, we added $\mathrm{ABs}(30 \mu \mathrm{g} / \mathrm{ml})$ into the culture medium (without BMDMs) and put the medium in a humidified atmosphere of $5 \% \mathrm{CO}_{2}$. Then, the medium was collected $24 \mathrm{~h}$ later. The concentrations of IL10 and TGF- $\beta$ in the conditioned medium were measured by using murine ELISA kits (NeoBioscience, China) according to the protocols described by the manufacturers.

\section{TUNEL staining}

TdT-mediated dUTP nick-end labeling (TUNEL) staining was performed as manufacturers' protocols with the in situ cell death detection kit in frozen sections (Roche, Switzerland). Briefly, approximately $10-\mu \mathrm{m}$-thick sections were prepared from the skin tissues. After sequential pretreatment, each section was stained with TUNEL reagents for $1.5 \mathrm{~h}$ at $37^{\circ} \mathrm{C}$ in the dark environment and Hoechst 33342 was used to counterstain the nuclei. Digital photographs were taken by a confocal microscope (Olympus, Japan).

Table 1 Primer sequences

\begin{tabular}{lll}
\hline Gene & Forward & Reverse \\
\hline Gapdh & 5'-TGTGTCCGTCGTGGATCTGA-3' & 5'-TTGCTGTTGAAGTCGCAGGAG-3' \\
II-10 & 5'-GCCAGAGCCACATGCTCCTA-3' & 5'-GATAAGGCTTGGCAACCCAAGTAA-3' \\
Arg-1 & 5'-AGCTCTGGGAATCTGCATGG-3' & 5'-ATGTACACGATGTCTITGGCAGATA-3' \\
Tgf- $\beta$ & 5'-CAAGCTGAACTTGA & 5'-TTTACTCAGTGCCAGAAGCTGGA-3' \\
& GCGAGGA-3' & \\
\hline
\end{tabular}




\section{Western blots}

BMDMs were treated with $\mathrm{ABs}$ at $5 \mu \mathrm{g} / \mathrm{ml}, 10 \mu \mathrm{g} / \mathrm{ml}$, $20 \mu \mathrm{g} / \mathrm{ml}$, and $30 \mu \mathrm{g} / \mathrm{ml}$ respectively. The culture medium was discarded, and the proteins from BMDMs were extracted by using RIPA buffer with protease inhibitor (Beyotime, China) 24 h later. After being quantified by BCA assay, the proteins were loaded on sodium dodecyl sulfate-polyacrylamide (SDS) gels and transferred to polyvinylidene fluoride (PVDF) membranes (Millipore, USA). Then, the membranes were blocked with $5 \%$ BSA for $2 \mathrm{~h}$ at room temperature and incubated with primary antibodies overnight at $4{ }^{\circ} \mathrm{C}$. Finally, the membranes were incubated with peroxidase-conjugated secondary antibodies (CWBio, China) for $1 \mathrm{~h}$ at room temperature. The protein bands were detected by the imaging system (Tanon, China) and quantified by ImageJ software. Primary antibodies include GAPDH (1:1000) (CWBio, CW0100, monoclonal, China), ARG-1 (1:400) (Cell Signaling Technology, 93668, monoclonal, USA), and TGF- $\beta$ (1:500) (Abcam, ab215715, monoclonal, UK).

\section{Statistical analysis}

All the data was expressed as the mean \pm standard deviation (SD). Comparisons of two groups were performed by two-tailed Student's $t$ tests, and comparisons of multiple groups were performed by one-way analysis of variance (ANOVA) with Tukey correction by using the Statistical Program for Social Science software (IBM, Armonk, NY, USA). $P<0.05$ was considered as statistically significant. Data tables are presented in additional files summarizing the mean and standard deviation of the data obtained (Additional Tables 1-7).

\section{Results}

Transplanted MSCs promoted cutaneous wound healing after transplantation

Bone marrow mesenchymal stem cells (BMMSCs) were isolated from 8-week-old female WT C57BL/6 mice, and the second passage was used for characterization. We firstly analyzed the expression of surface markers by flow cytometry. The MSCs we isolated highly expressed CD29, CD90, CD105, CD146, and Sca-1, while hematopoietic markers like CD11b, CD34, and CD45 were not detected (Fig. 1a, b). Then, we examined the colony formation property by using colony-forming unit assays (Fig. 1c). Meanwhile, we detected the osteogenic and adipogenic differentiation property by ALP staining, Alizarin red staining, and Oil Red $\mathrm{O}$ staining, respectively (Fig. 1d-f). In order to verify the therapeutic efficacy of the transplanted MSCs, we established the mouse skin wound healing models and locally employed exogenous MSCs for treatment (Fig. 2a). MSCs were embedded in PF-127, a biocompatible, nontoxic, and thermo-reversible material, which has been widely applied in many studies $[18,19]$. With a proper concentration, PF-127 solution is a free-flowing liquid at room temperature, but forms a hydrogel at body temperature, which can stay safely on the wound area [20]. Meanwhile, PBS was used as a blank control and PF-127 was used alone to exclude the effect of the gel. Photographs of the wound area were taken at five different time points during the wound healing procedure, and the wound healing rate was calculated as well (Fig. 2b, c). As we expected, the group treated with transplanted MSCs healed the fastest among the three groups, while no significant differences were found in the wound healing rate of the other two groups (Fig. 2c). We then collected the skin samples at day 14 and further conducted a histological analysis to assess the result of skin regeneration. As shown by H\&E and Masson staining (Fig. 2e, f, Additional file 1A-B), MSCs significantly enhanced the effect on cutaneous wound healing compared to that observed in the PBS or Gel groups. In the MSC group, we observed smaller scar areas, less infiltration of inflammatory cells, and a more integrate cutaneous structure with newly formed epithelium, some hair follicles as well as better deposited and organized collagen. In addition, we detected the expression level of cytokeratin 14 by immunofluorescent staining (Fig. 2g). Cytokeratin 14 is a marker of keratinocytes, the levels of which present the quality of re-epithelization in cutaneous wound healing [21]. The fluorescence intensity was significantly higher in the MSC group, which implied better re-epithelization in this group than the other two groups. All these data demonstrated that the transplanted MSCs accelerated the cutaneous wound healing rate and enhanced the cutaneous regeneration. To explore apoptosis of transplanted MSCs in vivo, we labeled the cells with PKH26 and detected apoptosis by TdT-mediated dUTP nick-end labeling (TUNEL) staining $24 \mathrm{~h}$ later after transplantation (Fig. 2h). Numerous PKH26-labeled MSCs were also TUNEL positive, which means the transplanted MSCs underwent apoptosis after transplantation. In summary, the transplanted MSCs promoted cutaneous wound healing while underwent apoptosis in a short time.

\section{MSC-derived ABs promoted cutaneous wound healing}

ABs were isolated by using the optimized gradient centrifugation protocol and then underwent characterization (Fig. 3a). Firstly, we observed the morphology and size distribution of ABs by SEM and dynamic light scattering (DLS) analysis (Fig. 3b, c). Then, we detected the expression of $\mathrm{C} 1 \mathrm{q}$ and Annexin $\mathrm{V}$ in ABs by immunofluorescent staining (Fig. 3d). In addition, protein expression levels of caspase- 3 and cleaved caspase- 3 were detected by western blots (Fig. 3e). The results showed the ABs we used in this study were vesicles as approximately $1 \mu \mathrm{m}$ in size, with $\mathrm{C} 1 \mathrm{q}$ and Annexin V positive and cleaved caspase 3 expressed. 


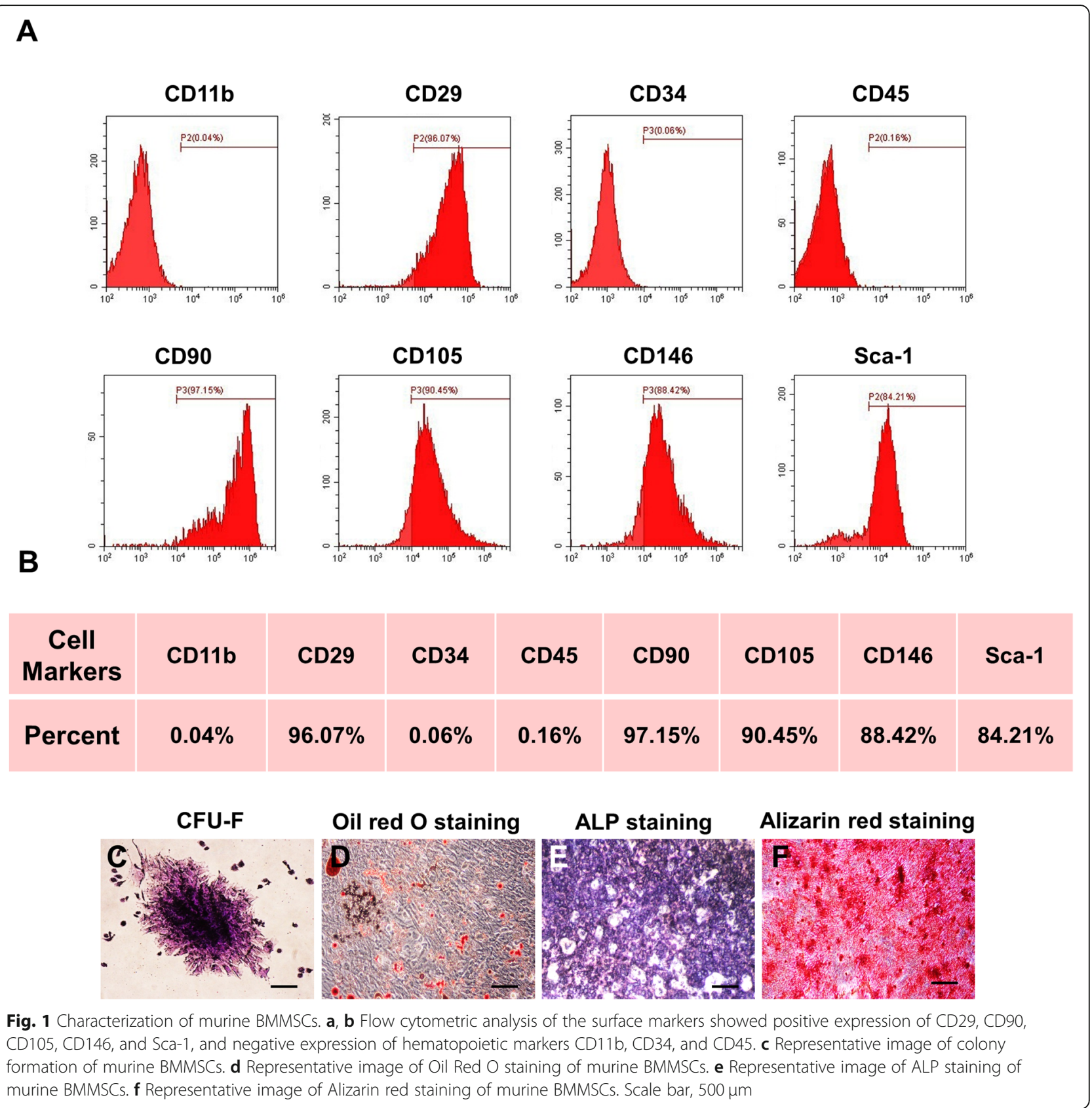

To investigate the therapeutic efficacy of ABs, we locally administrated $\mathrm{ABs}$ in mouse skin wound healing models by using the same methods with MSC-based therapy (Fig. 4a). The group treated with $\mathrm{ABs}$ healed the fastest among the three groups, while no significant differences were found in the wound healing rate compared with the other two groups (Fig. 4b, c). H\&E and Masson staining showed that ABs significantly enhanced cutaneous wound healing compared to that observed in the PBS or Gel groups. In the AB-loaded group, we observed smaller scar areas, less infiltration of inflammatory cells, and a more integrate cutaneous structure with newly formed epithelium, some hair follicles as well as better deposited and organized collagen (Fig. 4e-f, Additional file 2A-B). Immunofluorescent staining for cytokeratin14 showed the higher fluorescence intensity in the AB-loaded group (Fig. 4g). These data demonstrated that using $\mathrm{ABs}$ alone accelerated the cutaneous wound healing rate and enhanced the quality of skin regeneration. To confirm the fate of $\mathrm{ABs}$, we labeled $\mathrm{ABs}$ with PKH26 and traced them in vivo. We used F4/80 to mark macrophages, which is expressed on the cell membrane. As shown in Fig. 4h, the cells indicated by the arrow are F4/80 positive, which should be macrophages. ABs labeled with PKH26 are surrounded by the macrophages. Therefore, we 

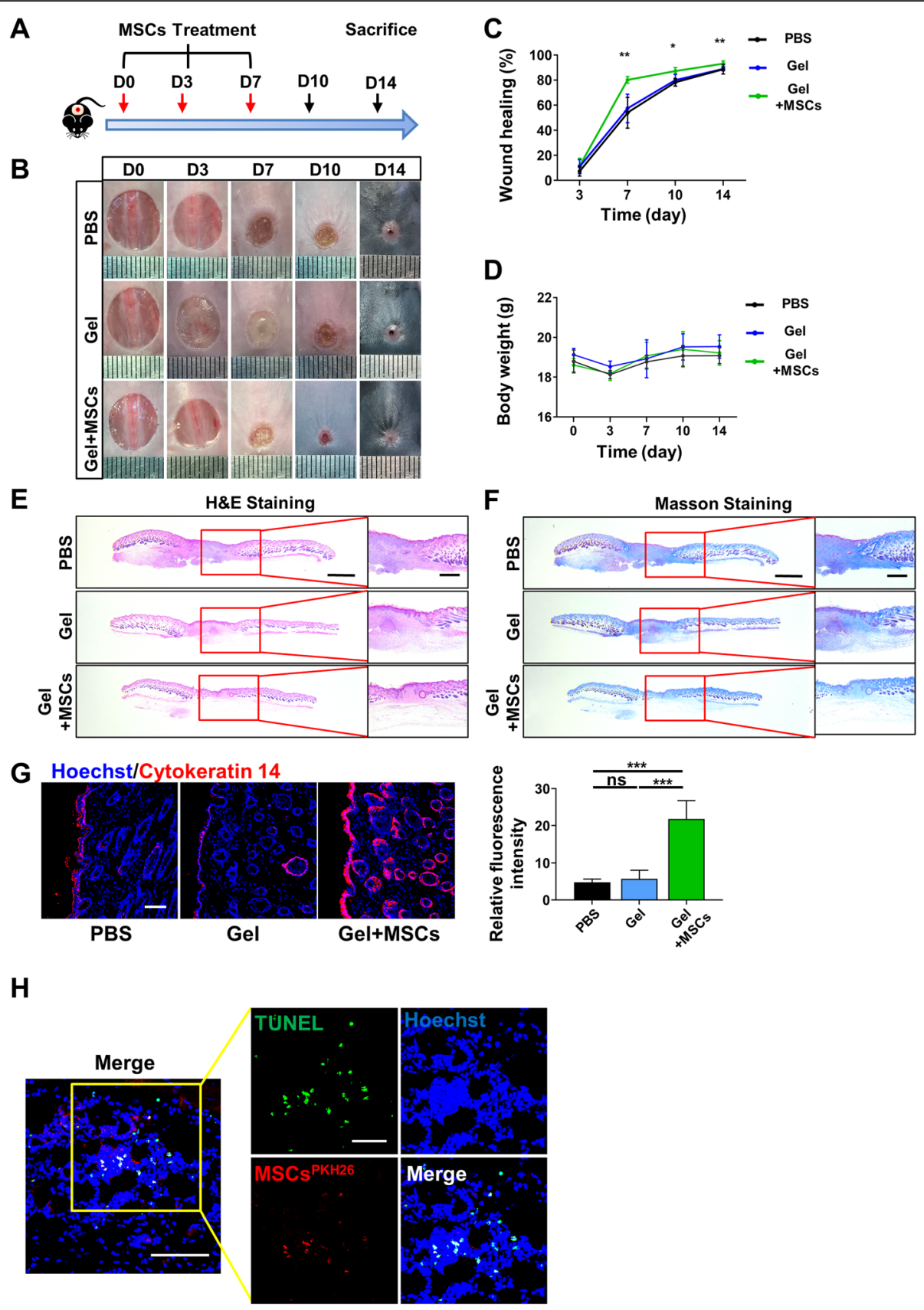

Fig. 2 Transplanted MSCs promoted cutaneous wound healing while underwent apoptosis. a The schematic graph shows the protocol of establishment and treatment for mouse skin wound models. b Representative photographs of cutaneous wounds in different groups at different time points during the wound healing procedure. c Quantification of the wound healing rate. $\mathbf{d}$ Quantification of the body weight. e Representative images of the H\&E staining of the skin samples. Scale bar, $1 \mathrm{~mm}$ in low-magnification images, $500 \mu \mathrm{m}$ in high-magnification images. $\mathbf{f}$ Representative images of the Masson staining of the skin samples. Scale bar, $1 \mathrm{~mm}$ in low-magnification images, $500 \mu \mathrm{m}$ in highmagnification images. $\mathbf{g}$ Representative images and quantification of the cytokeratin 14 expression in the skin samples. Scale bar, $100 \mu m$. $\mathbf{h}$ Representative images of TUNEL staining of skin tissue. Scale bar, $100 \mu \mathrm{m}$ in low-magnification images, $50 \mu \mathrm{m}$ in high-magnification images. $n=6$ per group. Data shown as mean \pm SD. ${ }^{*} P<0.05$; ${ }^{* *} P<0.01$; ${ }^{* *} P<0.001$; NS, not significant. PBS, phosphate-buffered saline; Gel, PF-127 gel; Gel+MSCs, MSCs embedded in PF-127; MSCs ${ }^{\text {PKH26, }}$, MSCs labeled with PKH26

estimated that $\mathrm{ABs}$ are probably engulfed by $\mathrm{F} 4 / 80$-positive macrophages $6 \mathrm{~h}$ after treatment. Since F4/80-positive macrophages were probably the target cells of $\mathrm{ABs}$, we next tested the effects of ABs on macrophages. Since M2 phenotypic macrophages are anti-inflammatory and play critical roles in tissue repair, we detected the expression levels of 


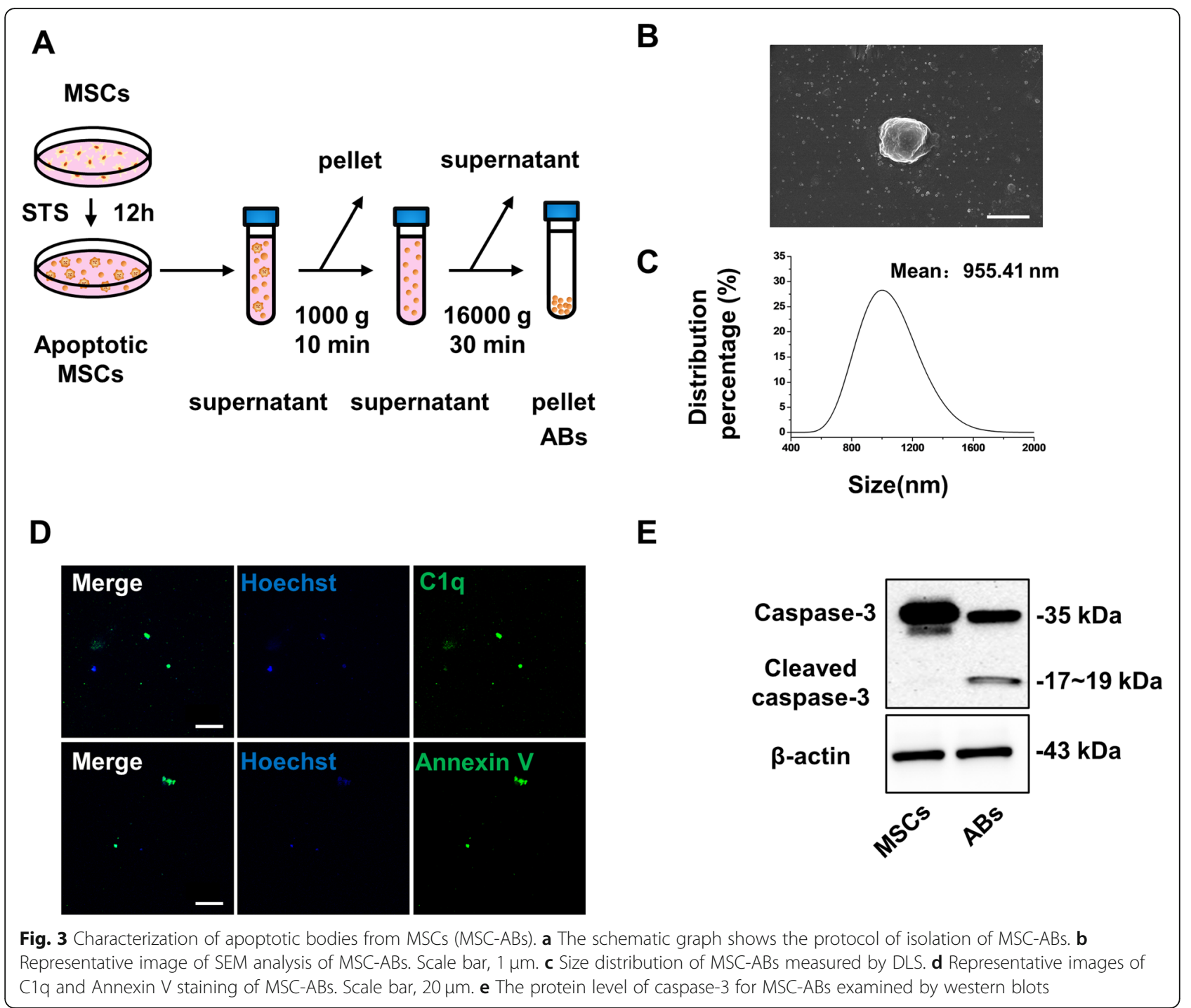

CD206, a marker of M2 phenotypic macrophages, by immunofluorescent staining (Fig. 4i). It is worth noting that CD206-positive cell numbers were much more in the ABloaded group than in the other two groups. The results showed that the group treated with $\mathrm{ABs}$ significantly expressed more CD206 than the other two groups 14 days after surgery. However, CD206 is also expressed in immature dendritic cells, which made it difficult to specify the changes of macrophages. To determine whether ABs has a direct effect on macrophage polarization, we next carried out several relevant experiments in vitro.

\section{MSC-derived ABs promoted polarization of macrophages} towards M2 phenotype

Since we observed that macrophages were probably the target cells of $\mathrm{ABs}$ in vivo and an increase number of CD206-positive cells, we further explored the effects of ABs on macrophage polarization in vitro. Macrophages are highly heterogeneous cells which present distinct properties fitting different organs and tissues [22]. In healthy skin, it is resident macrophages originating from the embryo that involve in homeostasis maintaining, while in damaged skin, circulating monocytes from the bone marrow are recruited into injury sites and differentiate into macrophages to aid clearance of damaged cells [23]. Firstly, we labeled ABs with PKH26 and then examined the phagocytosis of macrophages (Fig. 5a, b). The results showed that macrophages engulfed $\mathrm{ABs}$ in a time- and concentration-dependent way. Then, we used lipopolysaccharide (LPS) as a simple in vitro technically to mimic the inflammatory microenvironment for macrophages [16]. After stimulated by LPS, macrophages were treated with different concentrations of ABs and the unstimulated macrophages were used as control. We detected the expression levels of CD206 by immunofluorescent staining and found the decreased positive 

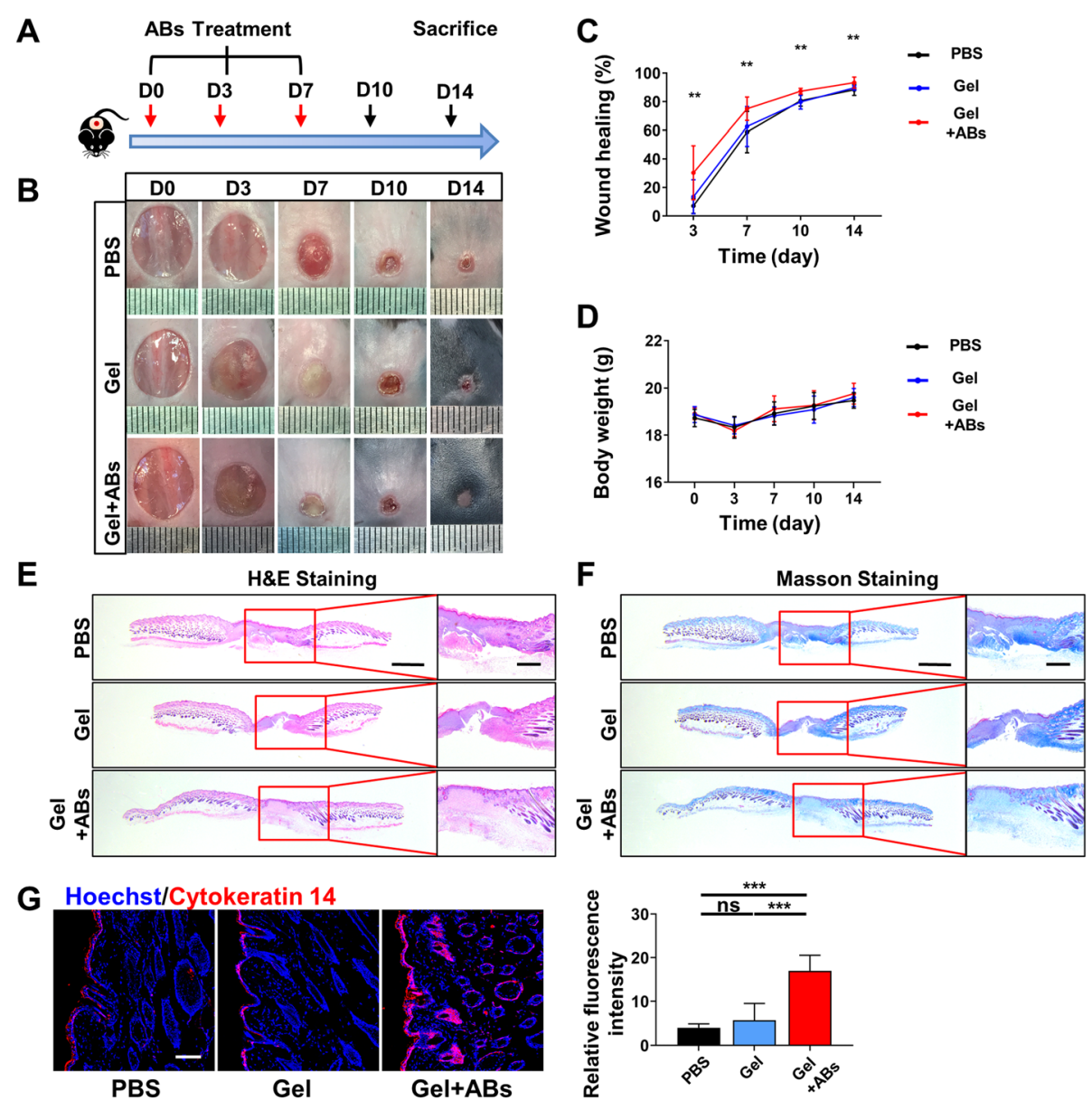

H Hoechst/ABs ${ }^{\mathrm{PKH} 26 / \mathrm{F} 4 / 80}$
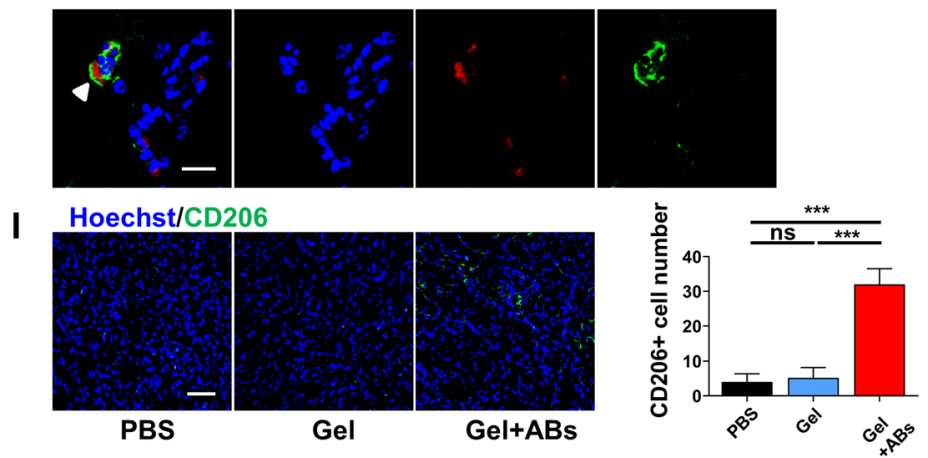

Fig. 4 ABs derived from MSCs promoted cutaneous wound healing. a The schematic graph shows the protocol of establishment and treatment for mouse skin wound models. $\mathbf{b}$ Representative photographs of cutaneous wounds in different groups at different time points during the wound healing procedure. c Quantification of the wound healing rate. $\mathbf{d}$ Quantification of the body weight. e Representative images of the H\&E staining of the skin samples. Scale bar, $1 \mathrm{~mm}$ in low-magnification images, $500 \mu \mathrm{m}$ in high-magnification images. $\mathbf{f}$ Representative images of the Masson staining of the skin samples. Scale bar, $1 \mathrm{~mm}$ in low-magnification images, $500 \mu \mathrm{m}$ in high-magnification images. $\mathbf{g}$ Representative images and quantification of the cytokeratin 14 expression in the skin tissue. Scale bar, $100 \mu \mathrm{m}$. $\mathbf{h}$ Immunofluorescence staining shows ABs engulfed by F4/80-positive macrophages in vivo. Scale bar, $20 \mu \mathrm{m}$. i Representative images and quantification of the CD206 expression in the skin tissue. Scale bar, $50 \mu \mathrm{m} . n=6$ per group. Data shown as mean \pm SD. ${ }^{*} P<0.05$; ${ }^{* *} P<0.01$; ${ }^{* * *} P<0.001$; NS, not significant. PBS, phosphate-buffered saline; Gel, PF-127 gel; Gel+ABs, ABs embedded in PF-127 gel; ABs ${ }^{\text {PKH26 }}$, ABs labeled with PKH26

cell number when stimulated by LPS. However, it was rescued by $\mathrm{ABs}$ in a concentration-dependent manner (Fig. 5c). Meanwhile, we collected supernatant of macrophages and investigated secretory factors like IL-10 and TGF- $\beta$ by ELISA (Fig. $5 \mathrm{~d}$, e). The secretion of both IL-10 and TGF- $\beta$ was increased by applying ABs. We also 

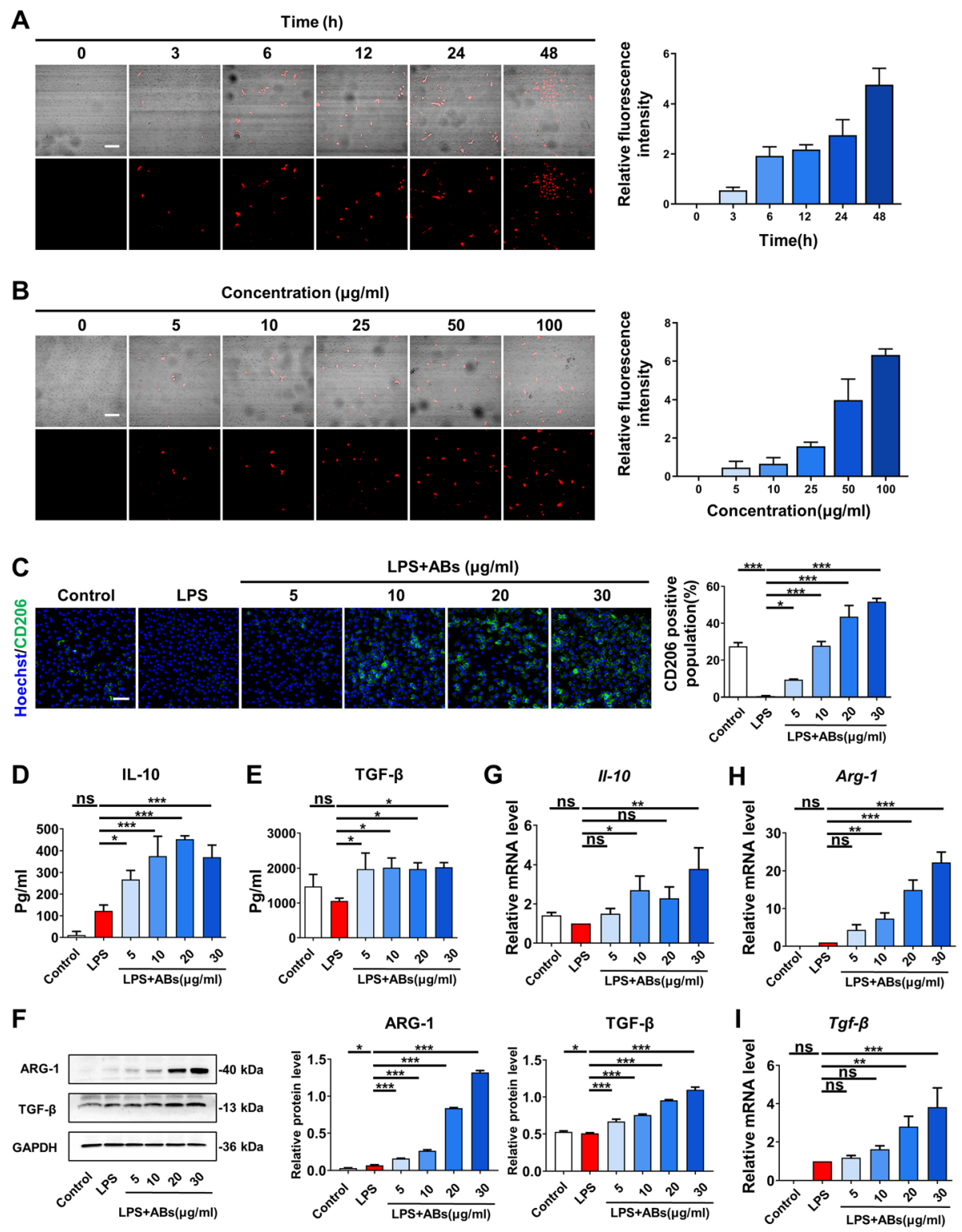

Fig. 5 ABs promoted macrophage polarization towards the M2 phenotype. a The immunofluorescence images of time-dependent uptake of ABs by macrophages. Scale bar, $100 \mu \mathrm{m}$. b The immunofluorescence images of concentration-dependent uptake of ABs by macrophages. Scale bar, $100 \mu \mathrm{m}$. c The immunofluorescence images and quantification of the CD206 expression level of macrophages treated with different concentrations of ABs. Scale bar, $100 \mu \mathrm{m}$. d, e The detection of secretory factors IL-10 (d) and TGF- $\beta$ (e) released by macrophages treated with different concentrations of ABs by ELISA. $\mathbf{f}$ The protein levels of ARG-1 and TGF- $\beta$ of macrophages treated with different concentrations of ABs examined by western blots. $\mathbf{g}-\mathbf{i}$ The gene expression level of II-10 (g), Arg-1 (h), and Tgf- $\beta$ (i) of macrophages treated with different concentrations of ABs examined by qPCR. $n=3$ per group. Data shown as mean \pm SD. ${ }^{*} P<0.05$; ${ }^{* *} P<0.01 ;{ }^{* *} P<0.001$; NS, not significant

investigated the secretion of both IL-10 and TGF- $\beta$ from ABs alone, and the results showed that the levels of both TGF- $\beta$ and IL- 10 released by ABs are almost undetectable, which demonstrated that macrophages are the source of the molecules (Additional file 3). Next, in the presence of both macrophages and $\mathrm{ABs}$, the expression levels of ARG-1 and TGF- $\beta$ were increased with concentrations of $\mathrm{ABs}$ detecting by western blots (Fig. 5f).
Meanwhile, in the presence of both macrophages and ABs, the expression levels of genes like Il-10, Arg-1, and $T g f-\beta$ were examined and they all expressed higher when treated by ABs (Fig. $5 \mathrm{~g}-\mathrm{i}$ ). Thus, these results indicated that $A B s$ promoted the conversion of macrophages towards the M2 phenotype, which serves as anti-inflammatory macrophages promoting cutaneous wound healing. 


\section{Macrophages treated by $A B s$ further promoted migration and proliferation of fibroblasts}

Besides macrophages, some other cell types also participate in the cutaneous wound healing process. Fibroblasts are the major component cell types of the dermis and contribute to the new skin tissue formation; we wondered whether $\mathrm{ABs}$ affected the function of fibroblasts. We firstly isolated fibroblasts from the skin and treated fibroblasts with different concentrations of ABs. After 24-h treatment, we examined the migration and proliferation abilities by scratch assay and Ki67 immunofluorescence staining, respectively (Fig. 6a, b). However, no significant increase was found in either the migration or the proliferation ability of fibroblasts. In addition to autologous functional transformation, communication between different cell types is also important in tissue repair. Considering the functional conversion of macrophages, we inferred that the converted macrophages further influenced the functions of fibroblasts. Then, we collected the conditioned medium of macrophages and treated fibroblasts with the conditioned medium. Interestingly, the conditioned medium from unstimulated and LPS-stimulated macrophages had no significant effect either on the migration or proliferation abilities of fibroblasts, while the conditioned medium from macrophages treated with $A B s$ enhanced both migration and proliferation abilities of fibroblasts (Fig. 6c, d). To sum up, ABs had no direct effects on fibroblasts whereas macrophages treated with $A B s$ improved both migration and proliferation abilities of fibroblasts, which promoted the cutaneous wound healing process synergistically.

\section{Discussion}

The skin serves as the first defensive line of the human body, so that it is one of the most vulnerable tissues [1]. Therefore, therapies for skin injuries have been highly focused. Among different treatments, stem cell-based therapy shows great potential and is the most popular candidate [2]. Mesenchymal stem cells (MSCs) are the kind of stem cells, which possess the capacity of selfrenewal and multi-directional differentiation [24]. MSCs can be isolated from multiple adult mesenchymal tissues like the bone marrow, adipose tissue, and so on [25]. Hence, in contrast with other stem cells, MSCs are much more available and less limited in ethics. Previous studies have demonstrated that exogenous MSCs had therapeutic effects on cutaneous wound healing in different models [26-29]. It has been thought that exogenous MSCs functioned in tissue regeneration through direct differentiation into component cell types of damaged tissue or releasing multiple cytokines and EVs to communicate with other cells via paracrine pathways $[7,25,30]$. Intriguingly, we found that large amounts of exogenous MSCs underwent apoptosis in a short time after transplantation which was consistent with our previous study [9].
Apoptosis is widely known as a manner of programmed cell death, which is not only of great importance in physiological processes like organogenesis and maintaining of tissue homeostasis, but also associated with pathological processes like tumorigenesis and tissue regeneration [11]. In the previous study, we demonstrated that apoptosis enhanced the inflammatory regulatory abilities of infused MSCs partially through activation of caspase-3 [9]. In addition to activation of effector caspase, apoptotic cells present several morphological changes including programmed degradation of DNA, chromatin condensation, cell shrinkage, and plasma blebbing [31]. Apoptotic bodies $(\mathrm{ABs})$ are referred to extracellular vesicles released during apoptosis [32]. As a type of EVs, ABs possess a membrane structure and carry diverse contents including organelles, proteins, mRNAs, and microRNAs [33]. A growing number of studies suggest that extracellular vesicles play an important role in cell-cell communications and functions as the vital mediator of cell signaling in multiple tissue regeneration [34, 35]. In this case, we extracted ABs derived from MSCs and validated the therapeutic effects of ABs in murine cutaneous wound healing models.

A great number of cells undergo apoptosis every day in the human body to ensure cell turnover and sustain tissue homeostasis. To avoid extra damage to the healthy tissue, it is necessary to remove the corpses of apoptotic cells efficiently [15]. There are two categories of phagocytes: nonprofessional phagocytes like epithelial cells and professional phagocytes like macrophages [12]. Notably, macrophages are the dominant phagocytes that take charge of recognizing and engulfing apoptotic cells or vesicles [14]. Thereby, we asked whether the ABs were mainly phagocytized by macrophages. We labeled the $\mathrm{ABs}$ and examined the phagocytosis by macrophages both in vivo and in vitro. As we inferred, ABs could be probably engulfed by macrophages. Classical cutaneous wound healing is a complicated process which includes three overlapping phases: hemostasis and inflammation, newly tissue formation, and tissue remodeling [36]. These phases are highly orchestrated by multiple cell types such as keratinocytes, fibroblasts, immune cells, and endothelial cells. In addition to scavenger cells, macrophages are also immune cells which play significant roles in balancing the inflammatory and repairing processes during the wound healing process [37]. Macrophages are sufficiently flexible cells which polarize to disparate phenotypes in order to adapt to the microenvironment [16]. Accumulating evidence suggests that MSCs and apoptotic cells can trigger the polarization of macrophages towards the M2 phenotype [38-40]. Now that ABs were generated by apoptotic MSCs and largely taken up by macrophages, we then tested the effects of $\mathrm{ABs}$ on the phenotypic switch and functional changes of macrophages. The in vitro results showed that ABs could promote polarization of macrophages towards the M2 phenotype. 


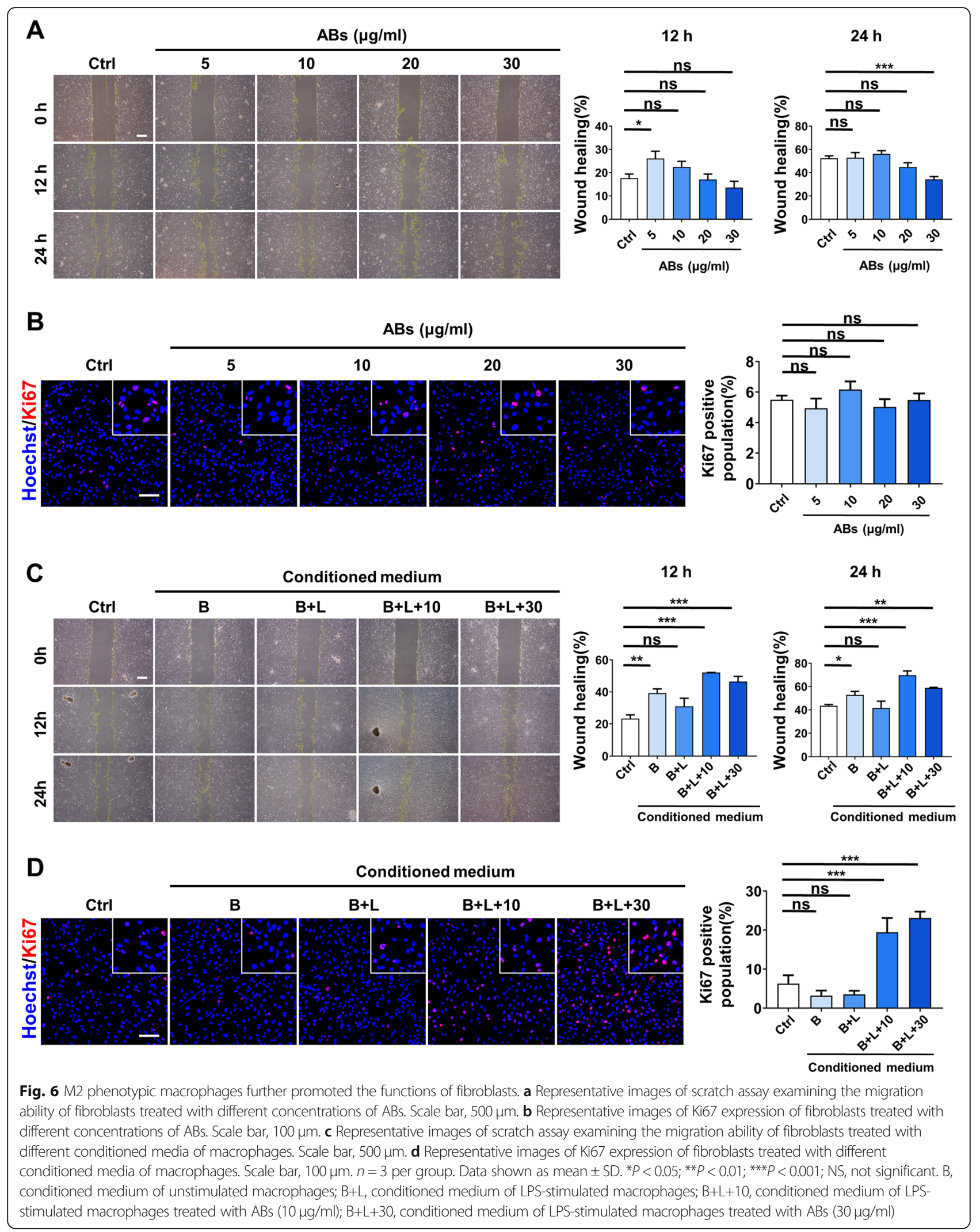


Combined with in vivo results, we inferred that $\mathrm{ABs}$ promote cutaneous wound healing at least partially via regulating macrophage polarization.

Since fibroblasts are the major components of the dermis and play important roles in newly tissue repair and tissue remodeling process [41], we wondered if ABs could also affect the functions of fibroblasts. Thus, we investigated the effects of ABs on proliferation and migration abilities of fibroblasts in vitro; however, no significant changes were found. We considered that as non-professional phagocytes, fibroblasts merely possess limited phagocytic abilities [12]. Therefore, unlike macrophages, fibroblasts may not engulf and handle large amounts of ABs. Nevertheless, it has been realized that communication between macrophages and fibroblasts is also a crucial factor in the tissue repair process [37]. Multiple soluble mediators released by macrophages have effects on functions of fibroblasts in the case of tissue repairing [12]. Hence, we further collected the conditioned medium from macrophages and examined the proliferation and migration abilities of fibroblasts. Interestingly, the conditioned medium from macrophages treated with $\mathrm{ABs}$ facilitated both proliferation and migration abilities of fibroblasts. We speculate that $\mathrm{ABs}$ facilitated the polarization of macrophages towards the M2 phenotype which release cytokines beneficial to the functions of fibroblasts. Taken together, we validated that rather than direct action, $\mathrm{ABs}$ can improve the capacity of fibroblasts indirectly through macrophages, which further contribute to the cutaneous wound healing process. In summary, the transplanted MSCs promote cutaneous wound healing partially via $A B s$ which formed during apoptosis. ABs are may largely engulfed by macrophages and thus trigger the polarization of macrophages towards the M2 phenotype. Furthermore, the educated macrophages enhance the functions of fibroblasts, which is also in favor of cutaneous wound healing.

\section{Conclusions}

In this study, we found that ABs accelerated the cutaneous wound healing process, and macrophages were probably the target cells of $\mathrm{ABs}$ in vivo. Through in vitro experiments, we proved that $\mathrm{ABs}$ promoted macrophage polarization towards the M2 phenotype, and M2 phenotype macrophages further enhanced the functions of fibroblasts, both of which were beneficial for cutaneous wound healing (Fig. 7). Collectively, our findings firstly elucidated that $A B s$ partially play an important role on the therapeutic effects of

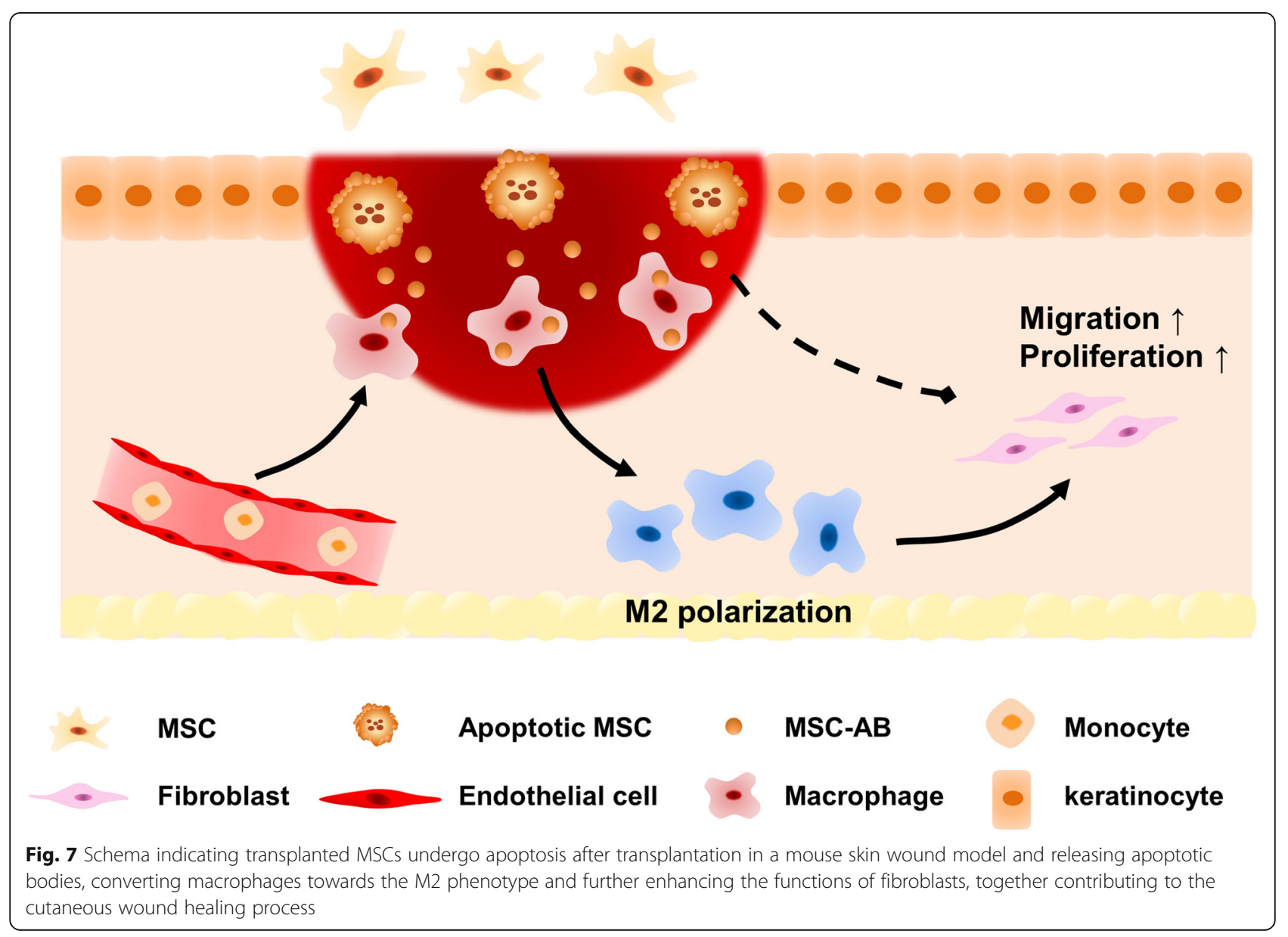


the administrated MSCs by promoting macrophage polarization. Meanwhile, considering the disadvantages and risks of MSC transplantation such as the low retention rate, safety issues, and the lack of cell sources, we may provide a new strategy for the treatment of cutaneous wound by utilizing ABs which derived from MSCs as a promising candidate to developing cell-free therapy.

\section{Supplementary Information}

The online version contains supplementary material available at https://doi. org/10.1186/s13287-020-02014-w.

Additional file 1. Images of histological staining of all the sections from all animals treated by MSCs. (A) Images of the H\&E staining of the skin samples. Scale bar, $1 \mathrm{~mm}$ in low magnification images, $500 \mu \mathrm{m}$ in high magnification images. (B) Images of the Masson staining of the skin samples. Scale bar, $1 \mathrm{~mm}$ in low magnification images, $500 \mu \mathrm{m}$ in high magnification images. PBS, phosphate buffer saline; Gel, PF-127 gel; Gel+MSCs, MSCs embedded in PF-127.

Additional file 2. Images of histological staining of all the sections from all animals treated by ABs. (A) Images of the H\&E staining of the skin samples. Scale bar, $1 \mathrm{~mm}$ in low magnification images, $500 \mu \mathrm{m}$ in high magnification images. (B) Images of the Masson staining of the skin samples. Scale bar, $1 \mathrm{~mm}$ in low magnification images, $500 \mu \mathrm{m}$ in high magnification images. PBS, phosphate buffer saline; Gel, PF-127 gel; Gel+ABs, MSC-ABs embedded in PF-127 gel.

Additional file 3. Excluding the source of molecules from ABs. The detection of secretory factors $\mathrm{LL}-10$ and TGF- $\beta$ released from $A B s$ alone by ELISA.

Additional file 4. Additional Tables 1-7 Data tables summarizing the mean and standard deviation of the data obtained.

\begin{abstract}
Abbreviations
MSCs: Mesenchymal stem cells; BMMSCs: Bone marrow mesenchymal stem cells; BMDM: Bone marrow-derived monocyte; EVs: Extracellular vesicles; ABs: Apoptotic bodies; MSC-ABs: MSC-derived ABs; ALP: Alkaline phosphatase; IL-10: Interleukin-10; TGF- $\beta$ : Transforming growth factor- $\beta$; Arg1: Arginase-1; a-MEM: Alpha-minimum essential medium; DMEM: Dulbecco's modified Eagle medium; FBS: Fetal bovine serum; BSA: Bovine serum albumin; M-CSF: Macrophage colony-stimulating factor;

LPS: Lipopolysaccharide; STS: Staurosporine; PF-127: Pluronic F-127; OCT: Optimal cutting temperature compound; SDS: Sodium dodecyl sulfatepolyacrylamide; PBS: Phosphate-buffered saline; SEM: Scanning electron microscopy; DLS: Dynamic light scattering; TUNEL: TdT-mediated dUTP nickend labeling
\end{abstract}

\section{Acknowledgements}

We would like to thank Fangfang Lin and Weihua Wang for their assistance with the sample collection and histological preparations.

\section{Authors' contributions}

J.L., X.Q., and Y. L. contributed equally to the study design, data analysis, data interpretation, and manuscript preparation. C.Z., G.D., and A.L. participated in the sample collection of partial animal experiments. Y.D., B.Z., J.Z., W.W., and S.L. participated in the data analysis and interpretation. S.L., B.G., and Y.J. conceived and supervised the study. All authors reviewed and approved the final version of the manuscript.

\section{Author's information}

Not applicable.

\section{Funding}

This research was funded by the National Key Research and Development Program of China (2016YFC1102900 to X.J., 2016 YFC1101400 to Y.J., 2018 YFB1 106902 to B.G.), the National Natural Science Foundation of China (31800817 to S.L., 31870970 to J.Z.), the Natural Science Basic Research
Program of Shaanxi (2018JM3026 to S.L.), the Young Elite Scientist Sponsorship Program by CAST (2017QNRC001 to S.L.), the Youth Program of National Natural Science Foundation of China (81601620 to B.Z.), and the Young Talent Support Program of Stomatology of FMMU (2018D4 to X.Q.).

\section{Availability of data and materials}

Not applicable.

\section{Ethics approval and consent to participate}

All experimental protocols were approved by the Animal Care Committee of the Fourth Military Medical University (FMMU), which was in accordance with the NIH Guide for the Care and Use of Laboratory Animals.

\section{Consent for publication}

Not applicable.

\section{Competing interests}

The authors declare that they have no competing interests.

\section{Author details}

${ }^{1}$ State Key Laboratory of Military Stomatology\& National Clinical Research Center for Oral Diseases \& Shaanxi International Joint Research Center for Oral Diseases, Center for Tissue Engineering, School of Stomatology, The Fourth Military Medical University, Xi'an 710032, Shaanxi, China. ${ }^{2}$ State Key Laboratory of Military Stomatology \& National Clinical Research Center for Oral Diseases \& Shaanxi Key Laboratory of Stomatology, Department of Prosthodontics, School of Stomatology, The Fourth Military Medical University, Xi'an 710032, Shaanxi, China. ${ }^{3}$ Department of Dermatology, Tangdu Hospital, The Fourth Military Medical University, Xi'an 710038, China. ${ }^{4}$ Department of Stomatology, General Hospital of Tibet Military Region, Lhasa 850007, Tibet, China. ${ }^{5}$ State Key Laboratory of Military Stomatology, National Clinical Research Center for Oral Diseases \& Shaanxi Key Laboratory of Oral Diseases, Department of Operative Dentistry and Endodontics, Fourth Military Medical University, Xi'an, China.

Received: 24 February 2020 Accepted: 3 November 2020

Published online: 27 November 2020

\section{References}

1. Miller KJ, Brown DA, Ibrahim MM, Ramchal TD, Levinson H. MicroRNAs in skin tissue engineering. Adv Drug Deliv Rev. 2015;88:16-36.

2. Lee DE, Ayoub N, Agrawal DK. Mesenchymal stem cells and cutaneous wound healing: novel methods to increase cell delivery and therapeutic efficacy. Stem Cell Res Ther. 2016;7:37.

3. Qi Y, Jiang D, Sindrilaru A, Stegemann A, Schatz S, Treiber N, et al. TSG-6 released from intradermally injected mesenchymal stem cells accelerates wound healing and reduces tissue fibrosis in murine full-thickness skin wounds. J Invest Dermatol. 2014;134(2):526-37.

4. Yates CC, Rodrigues M, Nuschke A, Johnson Zl, Whaley D, Stolz D, et al. Multipotent stromal cells/mesenchymal stem cells and fibroblasts combine to minimize skin hypertrophic scarring. Stem Cell Res Ther. 2017;8(1):193.

5. Pourjafar M, Saidijam M, Mansouri K, Ghasemibasir H, Karimi Dermani F, Najafi R. All-trans retinoic acid preconditioning enhances proliferation, angiogenesis and migration of mesenchymal stem cell in vitro and enhances wound repair in vivo. Cell Prolif. 2017;50(1):e12315.

6. Su N, Gao PL, Wang K, Wang JY, Zhong Y, Luo Y. Fibrous scaffolds potentiate the paracrine function of mesenchymal stem cells: a new dimension in cell-material interaction. Biomaterials. 2017;141:74-85.

7. Phinney DG, Pittenger MF. Concise review: MSC-derived exosomes for cellfree therapy. Stem Cells. 2017;35(4):851-8.

8. Ong HT, Dilley RJ. Novel non-angiogenic role for mesenchymal stem cellderived vascular endothelial growth factor on keratinocytes during wound healing. Cytokine Growth Factor Rev. 2018;44:69-79.

9. Liu S, Jiang L, Li H, Shi H, Luo H, Zhang Y, et al. Mesenchymal stem cells prevent hypertrophic scar formation via inflammatory regulation when undergoing apoptosis. J Invest Dermatol. 2014;134(10):2648-57.

10. Galleu A, Riffo-Vasquez Y, Trento C, Lomas C, Dolcetti L, Cheung TS, et al. Apoptosis in mesenchymal stromal cells induces in vivo recipient-mediated immunomodulation. Sci Transl Med.2017;9(416):eaam7828. 
11. Poon IK, Lucas CD, Rossi AG, Ravichandran KS. Apoptotic cell clearance: basic biology and therapeutic potential. Nat Rev Immunol. 2014;14(3): 166-80.

12. Gregory CD, Paterson M. An apoptosis-driven 'onco-regenerative niche': roles of tumour-associated macrophages and extracellular vesicles. Philos Trans R Soc Lond B Biol Sci. 2017;373(1737):20170003.

13. Gyorgy B, Szabo TG, Pasztoi M, Pal Z, Misjak P, Aradi B, et al. Membrane vesicles, current state-of-the-art: emerging role of extracellular vesicles. Cell Mol Life Sci. 2011;68(16):2667-88.

14. Ravichandran KS, Lorenz U. Engulfment of apoptotic cells: signals for a good meal. Nat Rev Immunol. 2007;7(12):964-74.

15. Elliott MR, Ravichandran KS. The dynamics of apoptotic cell clearance. Dev Cell. 2016;38(2):147-60.

16. Murray PJ. Macrophage polarization. Annu Rev Physiol. 2017;79:541-66.

17. Zhu H, Guo ZK, Jiang XX, Li H, Wang XY, Yao HY, et al. A protocol for isolation and culture of mesenchymal stem cells from mouse compact bone. Nat Protoc. 2010;5(3):550-60.

18. Lee J, Kim G. Three-dimensional hierarchical nanofibrous collagen scaffold fabricated using fibrillated collagen and pluronic F-127 for regenerating bone tissue. ACS Appl Mater Interfaces. 2018;10(42):35801-11.

19. Wu HF, Cen JS, Zhong Q, Chen L, Wang J, Deng DY, et al. The promotion of functional recovery and nerve regeneration after spinal cord injury by lentiviral vectors encoding Lingo-1 shRNA delivered by Pluronic F-127. Biomaterials. 2013;34(6):1686-700.

20. Alvarado-Gomez E, Martinez-Castanon G, Sanchez-Sanchez R, GanemRondero A, Yacaman MJ, Martinez-Gutierrez F. Evaluation of anti-biofilm and cytotoxic effect of a gel formulation with Pluronic F-127 and silver nanoparticles as a potential treatment for skin wounds. Mater Sci Eng C Mater Biol Appl. 2018;92:621-30.

21. Rousselle P, Braye F, Dayan G. Re-epithelialization of adult skin wounds: cellular mechanisms and therapeutic strategies. Adv Drug Deliv Rev. 2019; 146:344-65.

22. Mowat AM, Scott CL, Bain CC. Barrier-tissue macrophages: functional adaptation to environmental challenges. Nat Med. 2017:23(11):1258-70.

23. Malissen $B$, Tamoutounour $S$, Henri $S$. The origins and functions of dendritic cells and macrophages in the skin. Nat Rev Immunol. 2014;14(6):417-28.

24. Si YL, Zhao YL, Hao HJ, Fu XB, Han WD. MSCs: biological characteristics, clinical applications and their outstanding concerns. Ageing Res Rev. 2011; 10(1):93-103.

25. Galderisi U, Giordano A. The gap between the physiological and therapeutic roles of mesenchymal stem cells. Med Res Rev. 2014;34(5):1100-26.

26. Pelizzo G, Avanzini MA, Icaro Cornaglia A, Osti M, Romano P, Avolio L, et al. Mesenchymal stromal cells for cutaneous wound healing in a rabbit model: pre-clinical study applicable in the pediatric surgical setting. J Transl Med. 2015;13:219.

27. Murphy KC, Whitehead J, Falahee PC, Zhou D, Simon SI, Leach JK. Multifactorial experimental design to optimize the anti-inflammatory and proangiogenic potential of mesenchymal stem cell spheroids. Stem Cells. 2017:35(6):1493-504

28. Cappuzzello C, Doni A, Dander E, Pasqualini F, Nebuloni M, Bottazzi B, et al. Mesenchymal stromal cell-derived PTX3 promotes wound healing via fibrin remodeling. J Investig Dermatol. 2016;136(1):293-300.

29. Amini A, Pouriran R, Abdollahifar MA, Abbaszadeh HA, Ghoreishi SK, Chien $S$, et al. Stereological and molecular studies on the combined effects of photobiomodulation and human bone marrow mesenchymal stem cell conditioned medium on wound healing in diabetic rats. J Photochem Photobiol B. 2018;182:42-51.

30. Langrzyk A, Nowak WN, Stepniewski J, Jazwa A, Florczyk-Soluch U, Jozkowicz A, et al. Critical view on mesenchymal stromal cells in regenerative medicine. Antioxid Redox Signal. 2018;29(2):169-90.

31. Liu D, Kou X, Chen C, Liu S, Liu Y, Yu W, et al. Circulating apoptotic bodies maintain mesenchymal stem cell homeostasis and ameliorate osteopenia via transferring multiple cellular factors. Cell Res. 2018;28(9):918-33.

32. Brock CK, Wallin ST, Ruiz OE, Samms KM, Mandal A, Sumner EA, et al. Stem cell proliferation is induced by apoptotic bodies from dying cells during epithelial tissue maintenance. Nat Commun. 2019;10(1):1044.

33. Todorova D, Simoncini S, Lacroix R, Sabatier F, Dignat-George F. Extracellular vesicles in angiogenesis. Circ Res. 2017;120(10):1658-73.

34. Hodgkinson CP, Bareja A, Gomez JA, Dzau VJ. Emerging concepts in paracrine mechanisms in regenerative cardiovascular medicine and biology. Circ Res. 2016;118(1):95-107.
35. Rani S, Ritter T. The exosome - a naturally secreted nanoparticle and its application to wound healing. Adv Mater. 2016;28(27):5542-52.

36. Zhang S, Liu Y, Zhang X, Zhu D, Qi X, Cao X, et al. Prostaglandin E2 hydrogel improves cutaneous wound healing via M2 macrophages polarization. Theranostics. 2018;8(19):5348-61.

37. Wynn TA, Vannella KM. Macrophages in tissue repair, regeneration, and fibrosis. Immunity. 2016;44(3):450-62.

38. Weiss ARR, Dahlke MH. Immunomodulation by mesenchymal stem cells (MSCs): mechanisms of action of living, apoptotic, and dead MSCs. Front Immunol. 2019;10:1191.

39. Li P, Gong Z, Shultz LD, Ren G. Mesenchymal stem cells: from regeneration to cancer. Pharmacol Ther. 2019;200:42-54.

40. Morrison TJ, Jackson MV, Cunningham EK, Kissenpfennig A, McAuley DF, O'Kane CM, et al. Mesenchymal stromal cells modulate macrophages in clinically relevant lung injury models by extracellular vesicle mitochondrial transfer. Am J Respir Crit Care Med. 2017;196(10):1275-86.

41. Driskell RR, Watt FM. Understanding fibroblast heterogeneity in the skin. Trends Cell Biol. 2015;25(2):92-9.

\section{Publisher's Note}

Springer Nature remains neutral with regard to jurisdictional claims in published maps and institutional affiliations.
Ready to submit your research? Choose BMC and benefit from:

- fast, convenient online submission

- thorough peer review by experienced researchers in your field

- rapid publication on acceptance

- support for research data, including large and complex data types

- gold Open Access which fosters wider collaboration and increased citations

- maximum visibility for your research: over $100 \mathrm{M}$ website views per year

At $\mathrm{BMC}$, research is always in progress.

Learn more biomedcentral.com/submissions 